\section{Ocular perfusion pressure and choroidal thickness in eyes with polypoidal choroidal vasculopathy, wet-age-related macular degeneration, and normals}

P Rishi, E Rishi, G Mathur and V Raval
Shri Bhagwan Mahavir Vitreoretinal Services, Sankara Nethralya,

Chennai, India

Correspondence: Dr P Rishi, Shri Bhagwan Mahavir Vitreoretinal Services, Sankara Nethralya, 18, College Road, Nungambakkam, Chennai, Tamil Nadu 600006, India Tel: +91 442827 1616; Fax: +91442825 4180 E-mail: docrishi@yahoo. co.in

Received: 9 January 2013 Accepted in revised form: 23 April 2013

Published online: 14 June 2013

\begin{abstract}
Purpose To measure the choroidal thickness and ocular perfusion pressure in eyes with polypoidal choroidal vasculopathy (PCV), wet-age-related macular degeneration (AMD), and age-matched normal subjects, and look for a possible association between the two. Methods This was a prospective study including 22 eyes with PCV, 33 eyes with wet-AMD, and 35 age-matched normal eyes. Choroidal thickness was measured using enhanced depth imaging (EDI) with spectraldomain optical coherence tomography (SD OCT). Mean ocular perfusion pressure (MOPP) was calculated using the mathematical formula 2/3[DBP +1 / $3\{\mathrm{SBP} \times \mathrm{DBP}\}]-$ IOP $(\mathrm{DBP}-$ diastolic blood pressure, SBP-systolic blood pressure, IOP-intraocular pressure). Analyses were carried out using SPSS 14 software and comparisons of mean made using ${ }^{\prime} t^{\prime}$ tests. Results Eyes with PCV showed increased ( $285.9 \mu \mathrm{m}$; subfoveal) choroidal thickness, whereas eyes with wet-AMD $(119.4 \mu \mathrm{m}$; subfoveal) showed reduced choroidal thickness in comparison with normal eyes (186.77 $\mu \mathrm{m}$; subfoveal). MOPP in the PCV group was $57.85 \mathrm{~mm} \mathrm{Hg}$ ( $P$ value 0.00$)$, in the wet-AMD group was $52.1 \mathrm{~mm} \mathrm{Hg}$ ( $P$-value 0.12 ), and in the normal group was $49.79 \mathrm{~mm} \mathrm{Hg}$. Conclusion It is postulated that higher MOPP in eyes with PCV could have an etiologic implication in disease manifestation
\end{abstract}

and progression. Larger studies with longer follow-up may help validate these findings. Eye (2013) 27, 1038-1043; doi:10.1038/eye.2013.106; published online 14 June 2013

Keywords: ocular perfusion; choroid; optical coherence tomography; polypoidal choroidal vasculopathy; age-related macular degeneration; enhanced depth imaging

Introduction

Age-related macular degeneration (AMD) is the leading cause of blindness in the population over 60 years of age. ${ }^{1-5}$ Polypoidal choroidal vasculopathy $(\mathrm{PCV})$ is a disease entity that shares some clinical features similar to wetAMD. ${ }^{6-9}$ Yet, characteristic differences exist between the two entities in terms of pathogenesis, clinical features, and natural history. Progression is slower in PCV as compared with wet-AMD, scarring is less, and histopathological changes in eyes with PCV differ from those of choroidal neovascularization (CNV) due to AMD. Although the pathogenic mechanisms of PCV are still obscure, its associations with diabetes mellitus, ${ }^{10}$ hypertension, ${ }^{11}$ and thrombocytopenia ${ }^{12,13}$ have been reported. Hypertensive insult to the choroidal vascular bed has been described as a possible contributory factor in the development of PCV lesions. ${ }^{11}$ As the blood flow in an organ at any 
time is dictated by the perfusion pressure, ocular perfusion could have an important role in the pathogenesis of both wet-AMD and PCV.

The ability to visualize the choroid with enhanced depth imaging (EDI) using spectral domain optical coherence tomography (SD-OCT) has an important role in demonstrating the structural changes in the choroid and retina in normal and diseased states. ${ }^{14-20}$ In this study, we compared the mean choroidal thickness in eyes with PCV, wet-AMD, and age-matched normal subjects. We also recorded the mean ocular perfusion pressure (MOPP) in all eyes. The objective was to see if the structural changes in the choroid correlated with differences in MOPP. As MOPP is a potentially modifiable factor, investigating its role in relationship with PCV and wet-AMD could help in better understanding of the underlying disease mechanisms. To the best of our knowledge, the role of MOPP in relation to PCV and/or wet-AMD remains unclear.

\section{Materials and methods}

This was a single-centre, prospective, observational study. Institutional Review board (IRB) approval was obtained prior to starting the study. A written informed consent was obtained from all participating subjects. The study adhered to the tenets of the Helsinki declaration. We certify that all applicable institutional and governmental regulations concerning the ethical use of human volunteers were followed during this research. Patients included were diagnosed cases of PCV, wet AMD, and age-matched normal subjects. Subjects underwent comprehensive ophthalmological examination that included best-corrected visual acuity, slit lamp biomicroscopy, fundus fluorescein angiography (FFA), ICGA (FF 450 Fundus Camera, Carl Zeiss Meditec Inc., Dublin, CA, USA), and SD OCT (Cirrus OCT, Carl Zeiss Meditec Inc.). PCV was defined as clinical and angiographic findings of branching vascular network and terminating polypoidal lesion(s). Diagnosis of wetAMD was also based on a combination of clinical findings, FFA, and ICGA to exclude polyps or poypoidal $\mathrm{CNV}$. In patients with both eyes having the disease, only one eye was included to avoid the age bias from skewing the data. Normal subjects that were included in the study were drawn from attendants of the patients in the clinic. The normal subjects were screened for retinal and systemic diseases. Exclusion criteria included the presence of refractive errors more than $\pm 3.0 \mathrm{D}$, significant media opacities, dense subfoveal hemorrhages, history of ocular inflammation, history of $\mathrm{RD}$, history of PDT, previous ocular surgery like vitrectomy, history of ocular trauma, and glaucoma. Eyes with history of treatment with intravitreal injections of triamcinolone acetate (IVTA) or anti-vascular endothelial growth factor (VEGF) agents within the last 3 months were excluded. All cases of wet-AMD and PCV included in the study had active disease.

\section{Choroidal thickness}

Choroidal thickness was measured using EDI with SDOCT (see Figure 1). All the measurements were taken manually by two independent observers. In case of disagreement, the first author (PR) chose from one of the two readings. The EDI SD-OCT allows acquisition of

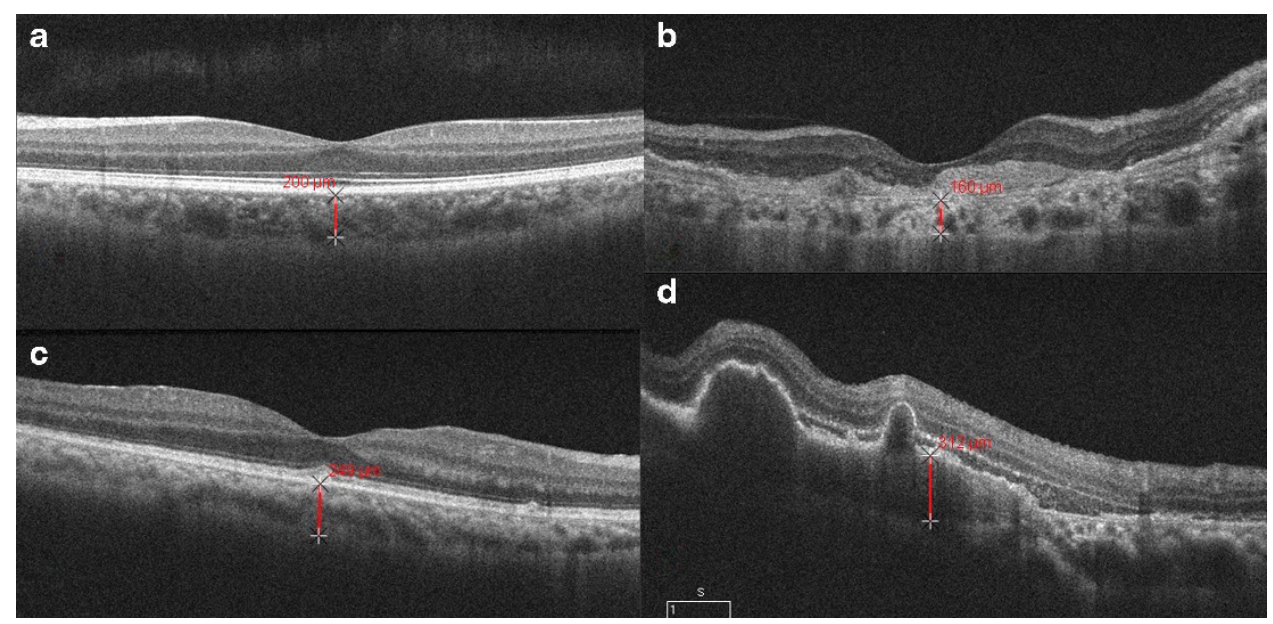

Figure 1 OCT scan on the left top (a) from a normal eye with a subfoveal choroidal thickness of $200 \mu \mathrm{m}$. OCT scan on the right top (b) from an eye with wet-AMD showing a high reflective subretinal, fusiform lesion suggestive of choroidal neovascularization. Subfoveal choroidal thickness measures $160 \mu \mathrm{m}$. OCT scan on the bottom left (c) is from an eye with PCV. Subfoveal choroidal thickness measures $249 \mu \mathrm{m}$. OCT scan on the bottom right (d) is from an eye with PCV showing polypoidal choroidal lesions with sublesional choroidal thickness measuring $312 \mu \mathrm{m}$. 
raster scans that are constructed from 20 B-scan images obtained at the same location. To improve visualization of the deeper (subretinal) structures, the SD-OCT is placed close enough to the eye to obtain an inverted image. The measurements were taken at the fovea and at $500-\mu \mathrm{m}$ intervals nasally, temporally, superiorly, and inferiorly until a distance of $2500 \mu \mathrm{m}$ from the foveola. Choroidal thickness was measured from the base of the hyper-reflective RPE-Bruchs membrane layer to the 'choroid-scleral' junction. The measurements at 21 different points in each eye were obtained by two independent observers and compared. Affected and 'unaffected' fellow eyes were compared for the PCV and wet-AMD groups.

\section{Blood pressure}

Blood pressure was recorded in the right arm in the sitting position after $5 \mathrm{~min}$ of rest, to the nearest $2 \mathrm{~mm} \mathrm{Hg}$ using a mercury sphygmomanometer (Diamond Deluxe BP apparatus, Pune, India), according to a protocol similar to that used in the Multi-ethnic Study of Atherosclerosis. ${ }^{21}$ Two readings were recorded, $5 \mathrm{~min}$ apart. A third measurement was made if the systolic blood pressure (SBP) differed by more than $10 \mathrm{~mm} \mathrm{Hg}$ or the diastolic blood pressure (DBP) differed by more than $5 \mathrm{~mm} \mathrm{Hg}{ }^{22}$ The mean between the two closest readings was taken as the blood pressure. Hypertension was defined as $\mathrm{SBP} \geq 140 \mathrm{~mm} \mathrm{Hg}$ or $\mathrm{DBP} \geq 90 \mathrm{~mm} \mathrm{Hg}$.

\section{Intraocular pressure}

IOP in both eyes was measured using the Goldmann applanation tonometer (Zeiss AT 030 Applanation Tonometer, Carl Zeiss, Jena, Germany), using 0.05\% proparacaine eye drops as topical anesthesia and $2 \%$ fluorescein to stain the tear film. IOP for each individual was corrected according to the central corneal thickness.

\section{Central corneal thickness}

The central corneal thickness was measured using Alcon ultrascan imaging system (Alcon Laboratories, Inc., Fort Worth, TX, USA).

\section{Ocular perfusion pressure}

Mean arterial pressure (MAP) was calculated as: $\mathrm{DBP}+1 / 3$ (SBP-DBP). MOPP was calculated using the formula, $\mathrm{MOPP}=2 / 3[\mathrm{DBP}+1 / 3\{\mathrm{SBP}-\mathrm{DBP}\}]-\mathrm{IOP}$.

All analyses were carried out using SPSS 14 software (Statistical Product and Service Solutions, IBM Corporation, Armonk, NY, USA) and comparisons of mean made using ' $t$ ' tests.

\section{Results}

Our study group included 30 eyes with PCV, 33 eyes with wet-AMD, and 35 eyes of normal subjects. There were 14 males and 16 females in the PCV group, 18 males and 15 females in the wet-AMD group, and 15 males and 20 females in the normal subjects. Mean age was $63.31 \pm 8.33$ years in the PCV group, $63.12 \pm 7.3$ years in the wet-AMD group, and $62.81 \pm 6.9$ years in the normal subjects (Table 1). There was no statistically significant difference in the mean ages between the normal and PCV group $(P=0.826)$, and between the normal and wetAMD group $(P=0.885)$. The sex ratio was comparative between the normal and PCV group $(P=0.67)$, and between the normal and wet-AMD group $(P=0.88)$. In the normal group, the mean subfoveal choroidal thickness was $186.77 \pm 11.38 \mu \mathrm{m}$, followed by decrease in choroidal thickness in the rest of the measured areas in the following order: temporal, superior, inferior, and nasal. A similar pattern of decreasing choroidal thickness was also observed in the PCV group and in the AMD group. Table 2 shows the mean choroidal thickness in eyes with PCV, eyes with wet-AMD, and eyes of agematched normal subjects. It also shows the mean

Table 1 Comparison of the means of variables in relation to the study groups

\begin{tabular}{lcccccc}
\hline & Age (years) & Systolic BP $(\mathrm{mm} \mathrm{Hg})$ & Diastolic BP $(\mathrm{mm} \mathrm{Hg})$ & MOPP $(\mathrm{mm} \mathrm{Hg})$ & $\mathrm{CCT}(\mu \mathrm{m})$ & $\mathrm{Mean} \mathrm{IOP}(\mathrm{mm} \mathrm{Hg})$ \\
\hline Mean PCV & $63.3 \pm 8.3$ & $145(128-170)$ & $88.7(80-96)$ & $57.9(45-67)$ & $465.4(430-475)$ & $14(10-15)$ \\
Mean normal & $62.8 \pm 6.9$ & $125(118-142)$ & $81.1(76-88)$ & $49.8(45-52)$ & $450(435-468.5)$ & $13.8(11-15)$ \\
$P$-value & 0.82 & 0 & 0.009 & 0 & 0.43 & 0.12 \\
Mean fellow PCV eye & $63.1 \pm 7.9$ & 144 & $86(79-90)$ & $55.5(42-58)$ & $465.6(444-478)$ & $14.8(12-16)$ \\
Mean normal & $62.8 \pm 6.9$ & $125(118-142)$ & $81.1(76-88)$ & $49.8(45-52)$ & $450(440-465)$ & $13.8(11-15)$ \\
$P$ value & 0.84 & 0.01 & 0.02 & 0.01 & 0 & 0.12 \\
Mean wet-AMD & $63.1 \pm 7.3$ & $130(110-146)$ & $80.33(80-92)$ & $52.1(42-55)$ & $456.8(435-469)$ & $14.6(13-15)$ \\
Mean normal & $62.8 \pm 6.9$ & $125(118-142)$ & $81.1(76-88)$ & $49.8(45-52)$ & $450(443-458)$ & $13.8(12-15)$ \\
$P$-value & 0.88 & 0.1 & 0.62 & 0.12 & 0.7 & 0.14 \\
\hline
\end{tabular}

Abbreviations: AMD, age-related macular degeneration; BP, blood pressure; CCT, central corneal thickness; IOP, intraocular pressure; MOPP, mean ocular perfusion pressure; PCV, polypoidal choroidal vasculopathy. 


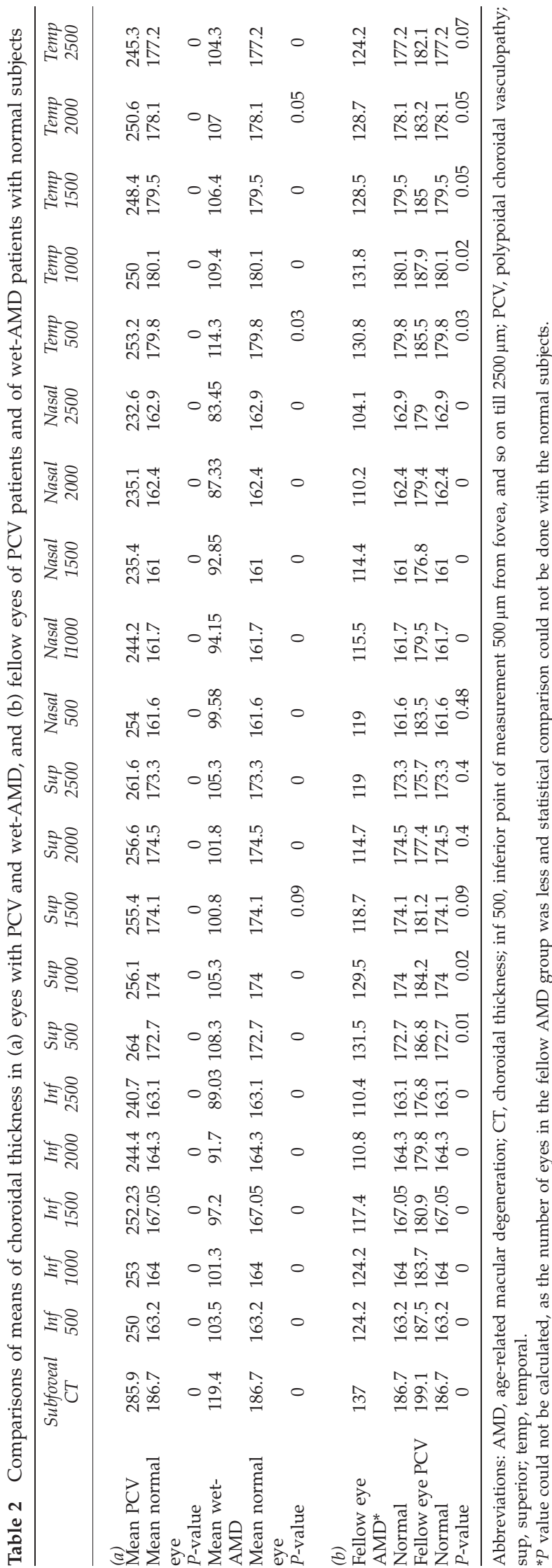

choroidal thickness in fellow (unaffected) eyes of patients with PCV or wet-AMD. In comparison with the normal group, choroidal thickness measurements at all the 21 points in each of the PCV patients showed a significantly higher choroidal thickness irrespective of the location of the PCV lesion. In the PCV group, 6 eyes had subfoveal polyp, 12 eyes had polyps superotemporal to fovea, 6 superonasal to fovea, 2 inferotemporal to fovea, 2 nasal to fovea, and 2 juxtapapillary. Mean choroidal thickness at all the 21 points measured in the wet-AMD eyes was significantly lower than the normal subjects. Among these, 15 eyes had subfoveal CNV, 5 had juxtafoveal CNV, and 2 had extrafoveal CNV. The fellow eyes of PCV and wet-AMD patients were also examined. Of the 15 fellow eyes of PCV patients evaluated, mean choroidal thickness was significantly higher at 18 of the 21 points in comparison with normal subjects. Of the 8 fellow eyes of wet-AMD patients evaluated, mean choroidal thickness at all 21 locations was lower than values from normal eyes, but statistical comparison could not be performed because of low numbers. The lower number included in the study was because either the fellow eyes were not normal on clinical examination (six eyes) or patients were not willing to get the evaluation done. A strong inter-observer correlation of choroidal thickness measurements in all eyes was found at each of the 21 locations measured along the horizontal and vertical scans of the macula (kappa 0.8). The correlation values of inter-observer measurements ranged from 1.00 to 1.01 .

The mean IOP (central corneal thickness-adjusted intraocular pressure) among the PCV group was $14 \pm 2 \mathrm{~mm} \mathrm{Hg}(P=0.16)$, among the wet-AMD group was $14.6 \pm 2.2 \mathrm{~mm} \mathrm{Hg}(P=0.18)$, and among the normal study group was $13.8 \pm 1.8 \mathrm{~mm} \mathrm{Hg}$. There was no significant difference in the intraocular pressure among the three groups. Sixteen of twenty-two PCV patients were known cases of systemic hypertension and were on medication for the same. The mean duration of systemic hypertension was 5.45 years (range: $4-8$ years) in the PCV group. Two patients had a history of diabetes mellitus and were on oral hypoglycemic agents. Eight of thirty-five patients in the the wet-AMD group were known cases of systemic hypertension with a mean duration of 4 years (range: 3-8 years). Among the normal study group, only 5 of the 35 patients were diagnosed cases of systemic hypertension, with the mean duration of hypertension being 4.5 years (range: $2-9$ years). The mean systolic blood pressure was $145 \mathrm{~mm} \mathrm{Hg}$ (range: $128-170 \mathrm{~mm} \mathrm{Hg}$ ) in the PCV group, $130 \mathrm{~mm} \mathrm{Hg}$ (range: $110-146 \mathrm{~mm} \mathrm{Hg}$ ) in the wet-AMD group, and $125 \mathrm{~mm} \mathrm{Hg}$ (range: $118-142 \mathrm{~mm} \mathrm{Hg}$ ) in normal subjects. The mean diastolic blood pressure was $88.77 \mathrm{~mm} \mathrm{Hg}$ 
(range: $80-96 \mathrm{~mm} \mathrm{Hg}$ ) in PCV patients, $80.33 \mathrm{~mm} \mathrm{Hg}$ (range: $80-92 \mathrm{~mm} \mathrm{Hg}$ ) in the wet-AMD group, and $81.13 \mathrm{~mm} \mathrm{Hg}$ (range: $76-88 \mathrm{~mm} \mathrm{Hg}$ ) in normal subjects (Table 1). MOPP in the PCV group was $57.85 \mathrm{~mm} \mathrm{Hg}$ (range: $45-67 \mathrm{~mm} \mathrm{Hg}$ ), that in the wet-AMD group was $52.1 \mathrm{~mm} \mathrm{Hg}$ (range: $42-55 \mathrm{~mm} \mathrm{Hg}$ ), and that in the normal study group $49.79 \mathrm{~mm} \mathrm{Hg}$ (range: $44-55 \mathrm{~mm} \mathrm{Hg}$ ) (Table 1). MOPP among the unaffected fellow eyes of patients with PCV was $55.45 \mathrm{~mm} \mathrm{Hg}$ (range: $46-62 \mathrm{~mm} \mathrm{Hg}$ ). Considering normal MOPP as $49 \mathrm{~mm} \mathrm{Hg}$ and that most of the values of MOPP among normal subjects fell within $1 \mathrm{SD}$, which was around $7 \mathrm{~mm} \mathrm{Hg}$, any value beyond $56 \mathrm{~mm} \mathrm{Hg}$ was considered to be as high. A chi-square nonparametric test was performed, which showed a significantly higher number of patients who had high MOPP in the PCV group compared with the AMD group $(P=0.00)$. A multivariate analysis was also performed to evaluate if MOPP was significantly higher in the PCV group compared with normal individuals, excluding the effects of IOP and blood pressure. The $P$-value resulted statistically significant $(P=0.043)$, indicating that MOPP is an independent risk factor in cases of PCV.

\section{Discussion}

The choroid in normal and diseased states has been investigated in a few studies with the help of a newly developed EDI technique using SD-OCT. The results for choroidal thickness in normal eyes and in the diseased eyes in our study are similar to those reported in the literature. ${ }^{23}$ However, we did not find any significant difference in relation to age $(P=0.826, P=0.885$, respectively) and refractive errors $(P=0.402, P=0.405$, respectively) among $\mathrm{PCV}$, wet-AMD, and normal subjects. ${ }^{23}$ In our study, we also included the fellow eyes of the same patients to see if any difference existed in the choroidal thickness as compared with normals. It was interesting to note that the mean choroidal thickness in the fellow eyes of PCV patients was also increased; the reason for increased choroidal thickness away from the PCV lesions is still debatable, possibly implying that both local and systemic risk factors have a role in disease manifestation. In contrast, mean choroidal thickness in wet-AMD eyes was significantly lower than in the normal group. The blood flow in an organ at any time is dictated or influenced by the perfusion pressure, which is defined as the difference between mean arterial blood pressure and venous pressure. The circumferential stress in a vessel is directly proportional to the perfusion pressure. ${ }^{24}$ A higher perfusion pressure can increase the circumferential stress damage to the vessel wall, leading to a continuing propensity to dilatation with subsequent hyperperfusion. ${ }^{25}$ Moreover, higher perfusion pressure and the resultant stress changes can also increase the net pressure gradient from vessels to tissue, leading to more fluid leaving the choroidal vessels (Starling's forces) and an increased risk for rupture (Laplace's law). ${ }^{26}$ Systemic hypertension has been described as a well-known risk factor for PCV. ${ }^{23,27-31}$ With less ${ }^{32}$ vascular structural support in the macular area, the choroid may also be predisposed to hypertensive insults. In our study, systemic hypertension was an associated factor in $55 \%$ of PCV patients compared with $11 \%$ in normals ( $P$-value 0.001). The mean systolic blood pressure in PCV eyes and unaffected fellow eyes was significantly higher than in normal ( $P=0.00$ and $P=0.01$, respectively) subjects. Similarly, the mean diastolic blood pressure was also significantly higher in PCV eyes and fellow unaffected eyes as compared with normals $(P=0.009$ and $P=0.02$, respectively). However, the mean systolic and diastolic blood pressures in wet-AMD eyes were similar to normal subjects $(P=0.1$ and $P=0.62$, respectively). MOPP was significantly high in PCV eyes $(P=0.00)$ and unaffected fellow eyes $(P=0.01)$ as compared with normals. On the other hand, MOPP in wet-AMD eyes was similar to normals $(P=0.12)$. One would suppose that a high MOPP value correlates with the clinical picture in eyes with PCV, having a suffused choroid. As systemic hypertension directly correlates with MOPP, high MOPP could possibly be one of the etiologic mechanisms for structural changes in the choroid in such eyes. Multivariate analysis showed that MOPP was also significantly higher in the PCV group when all other confounding factors like blood pressure and IOP were excluded. In this study, we found increased choroidal thickness not only in eyes having polypoidal lesions but also in the fellow eyes of the same patients. The increased choroidal thickness in fellow PCV eyes could possibly indicate the role of high MOPP in the etiopathogenesis of PCV, manifesting as serous detachments of RPE, with polypoidal lesions indicating dysregulation of local factors like autonomic input, venous stasis secondary to atherosclerotic changes in the vessel wall in that particular eye. The limitation in our study could be the diurnal variations in IOP that were not factored for. Though the IOP among the various groups was comparable, we do not expect significant variations in IOP, as none of the patients was a diagnosed case of glaucoma or a glaucoma suspect.

To conclude, choroidal thickness in eyes with PCV is higher than in normals and that in eyes with wet-AMD is lower. High MOPP in eyes with PCV could have an etiologic implication in disease manifestation and progression. Whether MOPP, which is modifiable with intensive control of systemic hypertension, could help modify the disease process requires further investigation. 
Summary

\section{What was known before}

- The choroid in normal and diseased states has been investigated in a few studies with the help of an EDI technique using SD-OCT.

\section{What this study adds}

- Choroidal thickness at points far away from the lesion in eyes with PCV showed significant increase in comparison with normals.

- Mean choroidal thickness in the fellow eyes of PCV patients showed significant increase in comparison with normals.

- Mean ocular perfusion pressure (MOPP) was significantly higher in the PCV group when other confounding factors like blood pressure and IOP were accounted for.

\section{Conflict of interest}

The authors declare no conflict of interest.

\section{References}

1 Yamada M, Hiratsuka Y, Roberts CB. Prevalence of visual impairment in the adult Japanese population by cause and severity and future projections. Ophthalmic Epidemiol 2010; 17: $50-57$.

2 Congdon N, O'Colmain B, Klaver CC. Causes and prevalence of visual impairment among adults in the United States. Arch Ophthalmol 2004; 122(4): 477-485.

3 Li Y, Xu L, Jonas JB, Yang H, Ma Y, Li J. Prevalence of agerelated maculopathy in the adult population in China: the Beijing eye study. Am J Ophthalmol 2006; 142(5): 788-3.

4 Seddon JM, Sharma S, Adelman RA. Evaluation of the clinical age-related maculopathy staging system. Ophthalmology 2006; 113: 260-266.

5 Age-Related Eye Disease Study Research Group. A simplified severity scale for age-related macular degeneration. Arch Ophthalmol 2005; 123: 1570-1574

6 Yannuzzi LA, Sorenson J, Spaide RF, Lipson B. Idiopathic polypoidal choroidal vasculopathy. Retina 1990; 10: 1-8.

7 Spaide RF, Yannuzzi LA, Slakter JS, Sorenson J, Orlach DA. Indocyanine green videoangiography of idiopathic polypoidal choroidal vasculopathy. Retina 1995; 15(2): 100-110.

8 Yannuzzi LA, Ciardella A, Spaide RF, Rabb M, Freund KB Orlock DA. The expanding clinical spectrum of idiopathic polypoidal choroidal vasculopathy. Arch Ophthalmol 1997; 115: 478-485.

9 Yannuzzi LA, Wong DWK, Storzolini BS, Goldbaum M, Tang KC, Spaide RF et al. Polypoidal choroidal vasculopathy and neovascularized age-related macular degeneration. Arch Ophthalmol 1999; 117: 1503-1510.

10 Rosa Jr, RH, Jl Davis, Eifrig CW. Clinicopathologic reports, case reports, and small case series: clinicopathologic correlation of idiopathic polypoidal choroidal vasculopathy. Arch Ophthalmol 2002; 120: 502-508.

11 Uyama M, Wada M, Nagai Y, Matsubara T, Matsunaga H, Fukushima I et al. Polypoidal choroidal vasculopathy: natural history. Am J Ophthalmol 2002; 133: 639-648.
12 Lip PL, Hope-Ross MW, Gibson JM. Idiopathic polypoidal choroidal vasculopathy: a disease with diverse clinical spectrum and systemic associations. Eye 2000; 14: 695-700.

13 Smith RE, Wise K, Kingsley RM. Idiopathic polypoidal choroidal vasculopathy and sickle cell retinopathy. Am J Ophthalmol 2000; 129: 544-546.

14 Spaide RF, Koizumi H, Pozzoni MC. Enhanced depth imaging spectral- domain optical coherence tomography. Am J Ophthalmol 2008; 146(4): 496-500.

15 Margolis R, Spaide RF. A pilot study of enhanced depth imaging optical coherence tomography of the choroid in normal Eyes. Am J Ophthalmol 2009; 147: 811-815.

16 Fujiwara T, Imamura Y, Margolis R, Slakter JS, Spaide RF Enhanced depth imaging optical coherence tomography of the choroid in highly myopic eyes. Am J Ophthalmol 2009; 148(3): 445-450.

17 Imamura Y, Fujiwara T, Margolis R, Spaide RF. Enhanced depth imaging optical coherence tomography of the choroids in central serous chorioretinopathy. Retina 2009; 29: 1469-1473.

18 Spaide RF. Enhanced depth imaging optical coherence tomography of retinal pigment epithelial detachment in age-related macular degeneration. Am J Ophthalmol 2009; 147: 644-652.

19 Manjunath V, Taha M, Fujimoto JG, Duker JS. Choroidal thickness in normal eyes measured using Cirrus Hd optical coherence tomography. Am J Ophthalmol 2010; 150: 325-329.

20 Ikuno Y, Kawaguchi K, Yasuno Y, Nouchi T. Choroidal thickness in healthy Japanese subjects. Invest Ophthalmol Vis Sci 2010; 51: 2173-2176.

21 Bild DE, Bluemke DA, Burke GL, Detrano R, Diez Roux AV, Folsom AR et al. Multi-ethnic study of atherosclerosis: objectives 316 and design. Am J Epidemiol 2002; 156: 871-881.

22 Wong TT, Wong TY, Foster PJ, Crowston JG, Fong CW, Aung T. SiMES Study Group. The relationship of intraocular pressure with age, systolic blood pressure, and central corneal thickness in an Asian population. Invest Ophthalmol vis Sci 2009; 50: 4097-4102.

23 Chung SE, Kang SW, Lee JH, Kim YT. Choroidal thickness in polypoidal choroidal vasculopathy and exudative age-related macular degeneration. Ophthalmology 2011; 118(5): 840-845.

24 Burton AC. Relation of structure to function of the tissues of the walls of blood vessels. Physiol Rev 1954; 34: 619-642.

25 Patel V, Rassam S, Newsom R, Wiek J, Kohner E. Retinal blood flow in diabetic retinopathy. BMJ 1992; 305: 678-683.

26 Guyton AC. Textbook of Medical Physiology. 7th edn. WB Saunders Co: Philadelphia, PA, USA, 1986; 393-409.

27 Nakashizuka H, Mitsumata M, Okisaka S, Shimada H, Kawamura A, Mori R et al. Clinicopathologic findings in polypoidal choroidal vasculopathy. Invest Ophthalmol Vis Sci 2008; 49(11): 4729-4737.

28 Leishman R. The eye in general vascular disease: hypertension and arteriosclerosis. Br J Ophthalmol 1957; 41: 641-701.

29 Manschot WA, Lee WR. Retinal neovascularisation arising from hyalinised blood vessels. Graefes Arch Clin Exp Ophthalmol 1984; 222: 63-70.

30 Lee WR, Blass GE, Shaw DC. Age-related retinal vasculopathy. Eye 1987; 1: 296-303.

31 Ross RD, Gitter KA, Cohen G, Schomaker KS. Idiopathic polypoidal choroidal vasculopathy associated with retinal arterial macroaneurysm and hypertensive retinopathy. Retina 1996; 16: 105-111.

32 Imamura Y, Engelbert M, Iida T, Freund KB, Yannuzzi LA. Polypoidal choroidal vasculopathy: a review. Surv Ophthalmol 2010; 55(6): 501-515. 